\title{
Realizing the Broker Based Dynamic Spectrum Allocation through LTE Virtualization and Uniform Auctioning
}

\author{
Yasir Zaki ${ }^{1}$, Manzoor Ahmed Khan ${ }^{2}$, Liang Zhao ${ }^{1}$, and Carmelita Görg ${ }^{1}$ \\ ${ }^{1}$ ComNets, University of Bremen, Germany \\ \{yzaki, zhaol, cg\}@comnets.uni-bremen. de \\ ${ }^{2}$ DAI-Labor/Technical University, Berlin, Germany \\ manzoor-ahmed. khan@dai-labor.de
}

\begin{abstract}
The next generation wireless networks are envisioned to follow the philosophy of buy when required, when it comes to the CApital EXpenditure (CAPEX) of operators over the infrastructure installation or extending new and dynamic resources. The dream of open market for new entrants and their coexistence with giant operators can be realized by the fancy concept of virtualization, which will be one of the key technologies in the future networks specially in the wireless part. Future virtualized mobile networks will comprise of a large number of (small) operators all competing for the spectrum resources. Such a scenario motivates and provisions the dynamic resource trading framework. This paper aims at presenting the realization concept of a dynamic spectrum trade market of future. We use the virtualized Long Term Evolution (LTE) as a realization framework for the proposed auctioned based dynamic spectrum sharing. We also investigate the profit function of spectrum broker and operators. We also study the realization of dynamic spectrum allocation on very small time instances (seconds) and investigate how the reservation price tunes the stake-holders' profit and resource allocation efficiency. The spectrum trade is based on the single auction multi-bid format and the paper further studies the impact of false bidding on the profit of spectrum broker (or network infrastructure provider).
\end{abstract}

Keywords: LTE, Wireless virtualization, Spectrum sharing, Uniform auctioning format.

\section{Introduction}

The scarce radio spectrum turns out to be the main pillar of the future wireless communications and spectrum management in any country is regulated by the a governmental body (e.g., FCC in USA or ECC in Europe). The current trend of spectrum allocation is derived by the concept of rigid frequency distribution through auctioning. Such allocations are static and specific to usage parameters (i.e., power, geographical scope etc.) and usage purposes (i.e., cellular communication, TV broadcasting, radio broadcasting etc.). Although auctions have been a success in this regard by putting essential spectrum in the hands of those who best value it and generating competition among the operators, such spectrum management may not cope up with the growing needs of spectrum with the user-centric network selection approach in place and the presence of various 
small scale new entrants e.g, MVNOs in the wireless market. We now briefly discuss the inefficiency introduced by the current fixed spectrum allocation market trends. The telecommunication operators bid for the amount of frequencies they are interested in, if declared winner, the bidding operators are allocated with some amount of frequencies for the periods spanning over years. This fact dictates that the operators frequency demands are the result of their peak traffic planning i.e., busy hour, which represents the peak network usage time. It should be noted that bandwidth demands are exposed to variation not only with respect to time (temporal variation) but also depends on the location (spatial variation). This, in a way addresses the issue of satisfying the operators' demands and reducing the call blocking at the operator end, however it causes temporal under-utilization in less busy periods. Hence the static spectrum allocation often leads to low spectrum utilization and results in fragmentation of the spectrum creating "white space" that cannot be used for either licensed or unlicensed services. The objective of improving spectrum utilization and providing more flexible spectrum management methods can be achieved by the currently well known concept, Dynamic Spectrum Allocation (DSA). DSA can significantly improve the spectrum utilization and provides a more flexible spectrum management method and promises much higher spectrum utilization efficiency. DSA concept brings a good news for the wireless service providers, as the flexible spectrum acquisition gives a particular provider the chance to easily adapt its system capacity to fit end users demand. In this paper, we confine our discussion more on dynamic spectrum allocation in user-centric paradigm, the interaction of stake-holders in the mentioned paradigm, and the technical realization framework for DSA. Obviously the operators' spectrum demand estimation turns out to be more more complicated in the mentioned configuration, we take care of this issue by carrying out number of simulations.

We take an opportunity here to justify the feasibility of dynamic spectrum allocation concept i.e., new generation radio interfaces support flexible transmission frequencies. The trend of future wireless technologies promises to ensure the user satisfaction for the envisioned dynamic bandwidth hungry service. e.g, Long Term Evolution (LTE) is expected to deliver five to ten times greater capacity than most current $3 \mathrm{G}$ networks with lower cost per bit. LTE also promises the flexible operational frequencies. Future wireless network communication is boosted by the concept of technology virtualization. When it comes to technical realization of the dynamic spectrum allocation concept, one of the attractive solutions is virtualization. Our choice of Virtualization as a technical solution is driven by the widespread and yet growing presence of this concept in the research literature e.g., many research projects including PlanetLab and GENI [1] [2] in the United States, AKARI [3] in Asia and 4WARD [4] in Europe. We now briefly comment on the proposed realization concept i.e., virtualization framework. There is a number of research activities in virtualization as well as a number of commercial solutions using virtualization: e.g., Server Virtualization, Router Virtualization, XEN, Cloud Computing etc. It was evidently obvious that the next step is to try bringing virtualization into the network as a whole and to combine all of the different virtualization research activities into forming what is known as "Virtual Networks" or "VNets".

Wireless virtualization is yet another very important aspect specially for the future. The best candidate for applying virtualization in the wireless domain is mobile 
networks. Mobile networks are the fastest growing networks globally and one of the biggest players in the future. In [5] it was shown that virtualization in mobile networks (represented by the Long Term Evolution (LTE)) has a number of advantages. Multiplexing gain as well as better overall resource utilization were the key gains achieved. In [6], a more practical framework was investigated for LTE virtualization and spectrum sharing among multiple virtual network operators. The framework focused on a contract based algorithm to share the spectrum between the operators.

\section{Related Work}

The concept of DSA first came up in the DARPA XG program [7], the project aims to develop, integrate, and evaluate the technology. The emphasis is on the enabling the user equipment that automatically selects spectrum and operating modes to both minimize distruption of existing users, and to ensure operation of U.S. systems. In [8], the authors propose a spectrum broker model that controls and provides operators the time bound access to a spectrum band. The authors investigated spectrum allocation algorithms for spectrum allocation in homogeneous CDMA networks and executed spectrum measurements in order to study the realizable spectrum gain that can be achieved using DSA.

The authors in [9] propose a scheme where the spectrum manager periodically allocates short-term spectrum licenses. The spectrum rights are traded amongs the operators for a fixed amount of time, the license for the allocated spectrum automatically expires after the predefined time period. However, [10] assumes that the operators follow the multi-unit auction format for the spectrum trade, where the sealed bids are submitted for the spectrum resources and the winner operator pays the second highest price (the price of resource is assumed to be charged on per unit basis). In [11], the authors present bidding framework, where the spectrum manager / broker tries to maximize its revenue, moreover, the authors claim that the proposed bidding framework is equally suitable fro heterogeneous channel and general complementary bidding function. The paper also presents greedy algorithms with the approximation bounds to solve the NP-hard allocation problems. The authors in [12] argue that the widely employed interference modeling (pairwise conflict graph) is weak and suggest the methods as to how to drive the interference model from the physical interference models so that it produces nearoptimal allocation. Zheng et al. [13] use Vickery Clark Groves (VCG) format of auction to model the spectrum allocation problem. A deviation from the use of distributed approaches is observed in authors centralized auction based approach [14], where authors assume pairwise interference conflict graph, piece-wise linear bidding functions, and homogeneous non-overlapping channels. Given these assumptions the authors authors formulate the allocation and pricing using the linear programming approach. Authors give the approximation bounds of the proposed heuristics and discuss the trade-off between revenue and fairness. They also argue on the difference between global marketclearing price and discriminatory pricing schemes. [15] relies on the VCG mechanism in a sealed-bid knapsack auction when determining spectrum allocation, but in the presented economic model the authors also account for the interaction between wireless service providers and users, and determine dynamic pricing rules to capture their conflict of interest. On the other hand they do not discuss interference issues. 


\section{Contribution}

This work concentrates on analyzing more realistic dynamic spectrum resource trade in future market. We focus on technical realization of the mentioned market and investigate market efficiency for different strategies of operators and network infrastructure provider. We explicitly model the operator, network infrastructure provider utility functions, operators valuation function and discuss the market behavior for scenarios with varying reservation values and bidding strategies. We thoroughly study and implement the technical framework based on virtualization of LTE access networks.

\section{Assumption}

We assume that the environment consists of an infrastructure or spectrum broker and various mobile network operators. We further assume that network operators do not own their own infrastructure, rather the infrastructure is traded between network operators and network infrastructure provider on a need basis. The payment made against the traded resources are based on the "pay-as-you-go" format. In order to capture the demand specific to different services, we differentiate the network operators with respect to service types i.e., each is characterized by mutually exclusive service types. The network operators assess their demands and periodically acquire the resource from the resource providers. Upon the demand realization, the resource providers take decision over resource allocation and the price per unit resource that it charges for the extended or rented resource.

\section{Spectrum (Infrastructure) Resource Trading}

We choose the uniform pricing auction to model the interaction at this stage, the motivation for choosing the mentioned auction format is the common use of this auctioning format in financial and other markets, which is evident by a large economic literature devoted to its study 1 . It is also argued that to a large extent, the FCC spectrum auctions can be viewed as a uniform-price auction [16]. In a uniform-price auction, small bidders can simply bid their valuations and be assured of paying only the market-clearing price [17]. The fact highlighed in [18] that in uniform price auctions the downstream playing field is level, in the sense that each licensee begins with the same foundational asset at the same price is also one of the motivating force to user the uniform price auctioning for spectrum trade. More on acution clearing algorithms can be found in [19]. In our formulation, the spectrum broker (virtualized LTE framework) is analogous to auctioneer, network operators are analogous to bidders, and the resource to be auctioned is analogous to auctioned-item. We assume that the auctioned-items are homogeneous and perfectly divisible. This assumption is strengthen by the fact that current trend of introducing flexibility in frequencies licensing i.e., providing operators with the technology neutral spectrum allocation. Let the distribution of auctioned item size has support in the range $\left[X_{\min }, X_{\max }\right]$, which defines the resource limits,

\footnotetext{
${ }^{1}$ Ofcom, Award of available spectrum: 1781.7-1785 MHz paired with 1876.7-1880 MHz: A Consultation, 16 September 2005.
} 
where $X_{\min }$ is a single PRB (Physical Resource Block) size and $X_{\max }$ is the total capacity of the spectrum resource, hereafter we use $C$ to represent the total resource capacity of the infrastructure provider. Let there are $N$ symmetric risk-neutral bidders (operators) who compete by simultaneously submitting their non-increasing demand functions $x_{i, k} \forall i \in N$, whereas the index $k$ is illustrated later in this section. These bidders have independent private valuation function of the auctioned item, which is driven by the bidders' demand and the service types. Although the resource is homogeneous, the bidders have different valuations for different amount of the resource. Such valuation is strictly influenced and is the consequence of the service types for which the resource is required. We assume that the market comprises of demands of two service types namely Guaranteed service and Non-guaranteed, intuitively the former has more strict resource requirements when compared with the later. Let the $\nu_{i}$ be the bidder private valuation of the auctioned item. Influenced by the comment given in the preceding sentence, the bidder valuation varies for demands of different service types, this is captured by the index $k$, thus the bidder valuation now can be represented by $\nu_{i, k}$ such that $\nu_{i, k} \neq \nu_{i, \tilde{k}} \forall k \neq \tilde{k}$. To illustrate this one has to consider the service demand patterns of the operators or putting it the other way operators spectrum valuation is driven by the operators' target market segment e.g., an operator targeting the fair users values the amount of spectrum demands for fair users more than the amount of spectrum for other user (service) types, the similar argument holds for vice versa. Thus the strategy for bidder $i \in N$ is non-increasing function $X_{i}:[0, \infty) \rightarrow\left[0, X_{\max }\right]$, and the his private valuation $\nu_{i, k}$, which is the evaluated spectrum price by the operator, the details of computing such valuation is given later in this section. Thus the operator bid is given by $b_{i, k, t}=\left\{x_{i, k}, \nu_{i, k}\right\}$. It should be noted that the valuation is computed as price per unit of the spectrum. We assume that the market behavior is represented by equation-1, and we term this market as spectrum trade market hereafter. As can be see that the demand curve is linear that expresses the demand as a linear function of the unit price. The choice of this market behavior is influence by its simplicity and wide

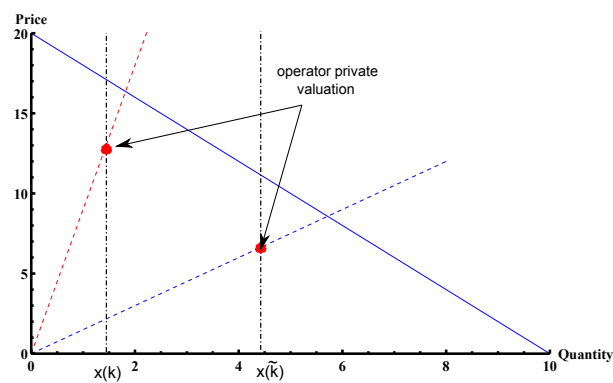

Fig. 1. Figure representing the valuation of the operators for different services presence in the literature.

$$
\pi(x):=-\zeta x+\beta,
$$

where $\zeta$ and $\beta$ are positives, the negative gradient represents the sensitivity of market towards the price, and $\beta$ represents the bound on price. $\pi$ represents the price. We know that the gradient introduces the ealsticity in the curve. However, the proposed problem formulation dictates an inelastic spectrum demand behavior i.e., irrespective of how price may change the demand remains the same. This is represented by a perpendicular to the quantity axis in Fig 1. Given such inelastic scenario, what about the operators' valuation computation? So far the valuation is the price value at the intersection of the demand perpendicular and normal negatively sloped (going down from left to right) 
linear demand curve. However, this does not capture the operator preference for different services. To address this issue, we introduce the operator valuation function. Thus now the operator valuation corresponds to the intersection of operator valuation function slope and the demand perpendicular. As depicted in Fig 1, we map the operator demands over the spectrum trade market. The operator's the valuation function, given by Equation 2 .

$$
\pi_{i, k}(x)=\frac{x_{i, k}}{\mu_{k}}
$$

where $\mu_{k}$ tunes the operators' valuation for the given demand and the service type such that; if the service type is of higher importance to the operator $\mu_{k}$ takes comparatively lower value than that of lower importance service. $\mu_{k}$ further can be translated as the function of number of operators in the spectrum competition and demands of service type i.e., real time service has higher value than that of background or non-real-time values i.e., $\mu(k, N-1)$. Although one may come up with any suitable $\mu($.$) function, in$ this work, we simply represent it by a real-value exposed to simple constraint of $\mu_{k, i} \neq$ $\mu_{\tilde{k}, i} \forall k \neq \tilde{k}$ and $\mu_{k, i} \neq \mu_{k, \tilde{i}} \forall i \neq \tilde{i}$. Furthermore, it should be noted from the Fig 1 that the price given by the intersection of the deman perpendicular and the negative slope of market curve is the upper bound on the prices set by the regulatory body.

Observation. In the problem under consideration, there are two interdependent markets; i) between operators and network infrastructure providers, termed as upstream market hereafter and ii) between operators and users, termed as downstream market hereafter. It should further be noted that downstream market demands (user service demands) influences the operator demands (operator resource demands). In this paper, the adopted methodology for estimating the downstream demands is based on the carried out simulation runs for all the virtual operators. In this connection we use the Exponential Moving Average (EMA) function to compute the estimated demands based on 20 seconds time intervals. Given the downstream demand, the upstream demand function can be computed.

Definition-1 - The operator valuation $\nu_{i, k}$ is directly translated in to $\pi_{i, k}$ i.e., based on service types and downstream market demands the operator reflects its valuation as $\pi_{i, k}$ such that $\nu_{k, u}=\pi_{i, k}$. Intuitively the false valuation $\tilde{\pi}_{i, k}$ is given by $\nu_{k, u} \neq \tilde{\pi}_{i, k}$.

Let $f$ be the mapping function that maps the downstream demands $Y$ over the upstream demand function $X$, such that $f(Y) \mapsto X$. For simplicity we assume that the mapping is bijective. Given the upstream demand function an operator computes its valuation of resource using Equation-2 Realizing the upstream demand and by Definition1 the operator formulates its bid for the resource, which is given by $b_{i}(X, \pi)$.

Allocation Rule. Given the uniform price auctioning format, let $\bar{b}$ represents the highest bid, and $p$ represents the stop out price, then the allocation rule is given by:

$$
a_{i}\left(X_{i}, b_{i}\right):=\left\{\begin{array}{l}
X_{i} \quad \text { if } \quad b_{i, k}=\bar{b} \wedge X_{i} \leq C \wedge b_{i, k}>p \\
\min \left\{X_{i}, C-\sum_{\tilde{b}_{-i} \in B, \tilde{b}_{-i} \backslash b_{i}} X_{\tilde{b}_{-i}}\right\} \quad \text { otherwise }
\end{array}\right.
$$


As can be seen from Equation 3 , the operator which is declared as the highest bidder gets the resources equivalent to its demands. In case the operator does not occupy the highest bidder position and resides in the winner list then it is allocated the residual resources not necessarily equivalent to its demands. The operator gets the residual demand when the infrastructure resource capacity is less than the operator demands(lower part of Equation-3). The resource allocated to each operator is independent of the auctioning format, however the payments do depend on the auction format.

Operator Utility Function. As we know that operators have different valuation of different amounts (i.e., spectrum for $k$ and $\tilde{k}$ type services) of spectrum. However, the allocation rule dictates that operators are allocated according to their aggregated demand request. Thus the operator profit function involves both spectrum amounts of different operator valuation values. We define the operator utility function for resource allocation $a_{i}$ to operator $i \in N$ and the stop-out price be $p$ as:

$$
u_{i}(a, p):=\left(\nu_{k}-p\right) x_{k}+\left(\nu_{\tilde{k}}-p\right) x_{\tilde{k}}
$$

As can be seen that operator utility increases in its valuation and demands and decreases in stop-out price.

Auctioneer utility function. The profit function or utility function of auctioneer (LTE virtualition framework) is the function of bidder demands and stop-out price and is given by: $u\left(\sum_{i \in N} X_{i}, p\right):=\sum_{\tilde{b} \in B} a_{\tilde{b}} \times\left(p-\frac{\sqrt{x}}{\lambda}\right)$ where $\frac{\sqrt{x}}{\lambda}$ represents the incurring cost of auctioneer, $\lambda$ is the controller that enables the auctioneer to scale the cost that follows the operator specific deployment pattern (i.e., co-location, site rentals, tower rental etc.), the detail modeling of cost function (modeling operational and maintenance, deployment costs etc.) is out of the scope of this paper. However, the choice of square-root function to capture the operator cost function is influenced by the continuous nature of the function for all non-negative numbers and differentiable for all positive numbers, the function also capture the realistic nature of the operator cost i.e., the operator initially incure more cost on improving service and such cost decreases with increase in demands. Spectrum broker maximizes its utility i.e., $\max _{p} u_{j}\left(\sum_{i \in N} X_{i}, p\right)$, which increases in $p$ and allocated resources and constrained by the operators' capacities. Thus the problem that the auctioneer solves is to decide the auction clearing or stop-out price and resource allocation (for allocation rule see Equation-3) i.e.,

$$
p=\sup \left\{p \mid \sum_{i \in N} X_{i} \geq K\right\}
$$

We also present the algorithm that is implemented by the auctioneer to take the decision over resource allocation and stop-out price as follows:

Lemma 1: In an homogeneous item uniform price auctions, the operators with multiple bids have a unique dominant strategy for each bid in different instances i.e., $b_{i, t_{1}}:=$ $\nu_{i, t_{1}, k}$ and $b_{i, t_{2}}:=\min \left\{R, \nu_{i, t_{2}, \tilde{k}}\right\}$

The proof is omitted due to space limitation. 


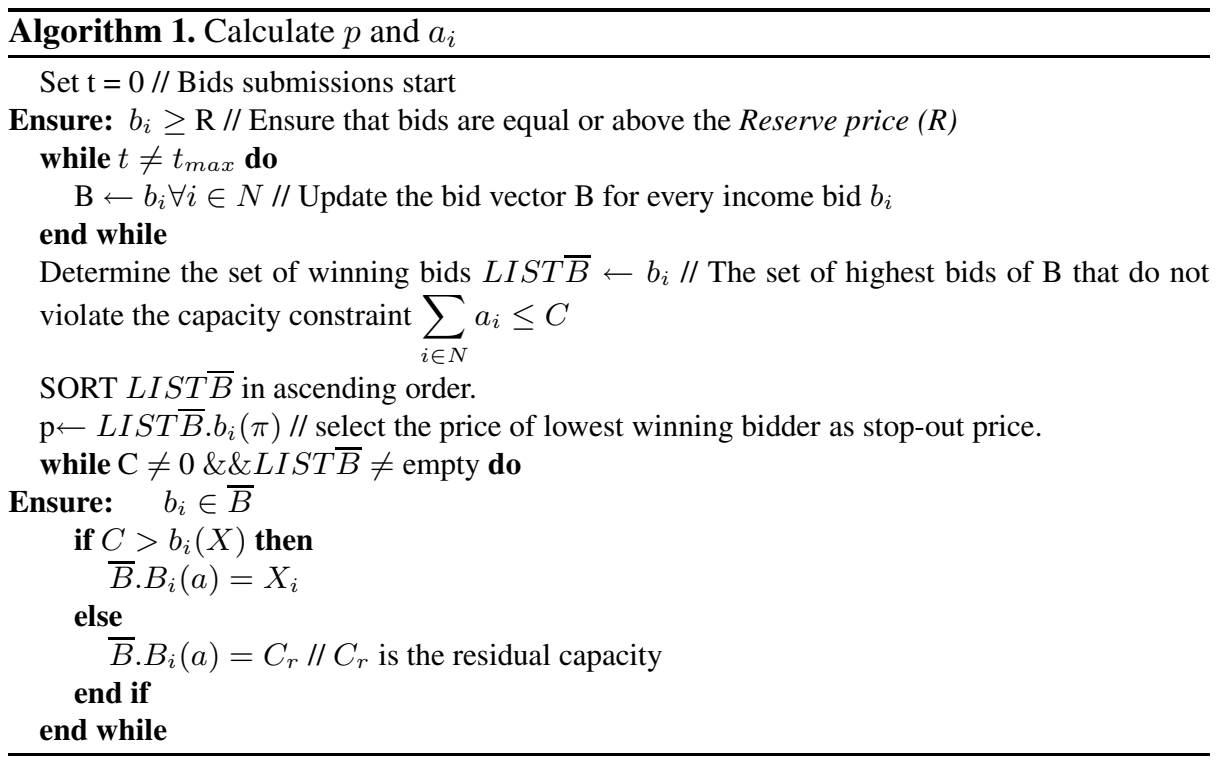

\subsection{Sequence of Actions}

i) Each bidder observes the demands (based on EMA, mentioned earlier), ii) Infrastructure provider announces the start of auction time $t_{\text {init }}$ and duration of bid submission i.e., $t_{\text {init }}-t_{\max }$, iii) Bidders submit their demands to the auctioneer at each per-unit price, which is the valuation of the bidder and attained from the Equation-2, iv) After the elapse of the submission time, the infrastructure provider observes the aggregated demand and sets the stop out price, which is equal or greater than the incurring cost over the unit resource. The auctioneer also decides each bidder's allocation, v) The allocation is executed through the hypervisor, vi) The process iterates over $i-i v$, after the inter-auction time $\delta t$ expires.

\section{Dynamic Spectrum Trade Realization Framework}

In this section, we present the LTE virtualization framework that we implement extensively to realize the proposed dynamic spectrum allocation concept. We virtualize the LTE network infrastructure (i.e., eNodeB, routers, ethernet links, and aGW etc.) so that multiple mobile network operators can create their own virtual network (depending on their requirements) on a common infrastructure. In the proposed virtualized network, we mainly foresee two different aspects; i) Physical infrastructure virtualization: virtualizing the LTE nodes and links and ii) Air interface virtualization: being able to virtualize the LTE spectrum. However, we in this paper focus on the later aspect, since virtualizing the air interface of the LTE system is a completely new concept and also the earlier aspect is extensively investigated in the research literature. 


\subsection{Air Interface Virtualization}

The eNodeB is the entity responsible for accessing the radio channel and scheduling the air interface resources between the users. The eNodeB has to be virtualized so as to virtualize the LTE air interface. Virtualizing the eNodeB is similar to node virtualization. The physical resource of the node (e.g., CPU, memory, I/O devices ... etc.) are shared between multiple virtual instances. XEN [20] is a well known PC virtualization solution that insert a layer called "Hypervisor" on top of the physical hardware to schedule the resources. From that, our LTE virtualization framework follows the same principle. A hypervisor is added on top of the PHY layer of the eNodeB, it is responsible for virtualizing the eNodeB node as well as the spectrum. The proposed framework can be seen in Figure-2

The architecture shows the physical eNodeB virtualized into a number of virtual eNodeBs. This is achieved by the hypervisor that sits on top of the physical resources of the eNodeB. In addition, the hypervisor is responsible for scheduling the spectrum, i.e., scheduling the air interface resources (OFDMA sub-carriers) between the virtual eNodeBs running on top. In the framework architecture two new entities should be highlighted: the

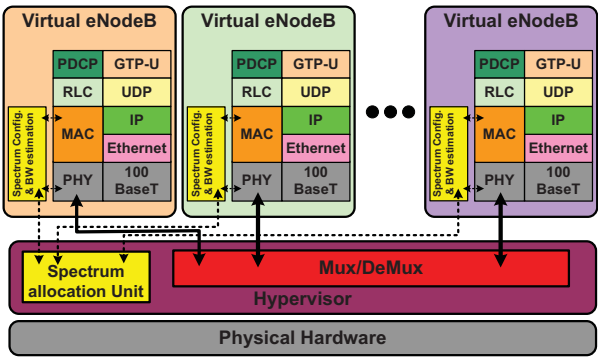

Fig. 2. LTE eNodeB virtualization framework architecture "Spectrum configuration and Bandwidth estimation" which is responsible for setting the spectrum the virtual eNodeB is supposed to operate in as well as estimating the required bandwidth of the operator. And the "Spectrum allocation unit" which is responsible for scheduling the spectrum among the different virtual eNodeBs. LTE uses OFDMA in the downlink, which means that the frequency band is divided into a number of sub-bands that are called Physical Resource Blocks (PRBs). A PRB is the smallest unit the LTE MAC scheduler can assign to a user. The Hypervisor schedule the PRBs between the different virtual operators, this process could be done by different mechanisms. In this paper, an auction based mechanism is used in the "Spectrum allocation unit" to auction the PRBs to the different virtual operators that bid for them.

\section{Simulation Model and Results Analysis}

The LTE virtualization simulation model is developed using OPNET [21] based on the 3GPP specifications. As explained earlier, the focus of the model is on the air interface virtualization and spectrum sharing between multiple virtual operators (all sharing the same eNodeB). Two scenarios are investigated based on the Auctioneer's (Infrastructure Provider) reserved price "“ $R$ ". The reserved price is the minimum price the Auctioneer is willing to sell the resources with, and any bid with a price lower than the reserved one will be rejected. The first scenario is configured no reserved price (i.e., $R=0$ ). The second scenario is configured with a dynamic reserved price that is a function of the 
Table 1. Simulation configurations

\begin{tabular}{l|l}
\hline \multicolumn{1}{c|}{ Parameter } & \multicolumn{1}{c}{ Assumption } \\
\hline \hline Number of virtual operators & 4 virtual operators with circular cells of 375 meters radius \\
\hline Total Number of PRBs (Spectrum) & 75 PRBs, i.e., about $15 \mathrm{MHz}$ \\
\hline Mobility model & Random Way Point (RWP) with vehicular speed (120 Km/h) \\
\hline $\begin{array}{l}\text { Number of active users } \\
\text { per virtual operator }\end{array}$ & VO1: $16 \mathrm{GBR}$ (video users) and 4 non-GBR (FTP users) \\
& VO2: $10 \mathrm{GBR}$ (video users) and 10 non-GBR (FTP users) \\
& VO3: $4 \mathrm{GBR}$ (video users) and 16 non-GBR (FTP users) \\
\hline VO1 price valuation & $\gamma 1=2$ and $\gamma 2=(2,4,6,8,10$ and 20$)$ \\
\hline VO2 price valuation & $\gamma 1=2$ and $\gamma 2=4$ \\
\hline VO3 price valuation & $\gamma 1=2$ and $\gamma 2=2$ \\
\hline Video traffic model & 24 frames per second with frame size $=1562$ Bytes \\
& Video call duration $=$ Exponential with 60 seconds mean \\
& Inter video call time $=$ Poison with 30 seconds mean \\
\hline FTP traffic model & FTP file size $=8 \mathrm{M}$ bytes \\
& Inter request time $=$ uniform between 50 and 75 seconds \\
\hline Auctioning parameters & Auction done every 20 seconds with $\gamma=0.25$ \\
\hline Simulation runtime & 1000 seconds \\
\hline
\end{tabular}

total resources demand, this is calculated as follows: $R=\gamma \cdot \sqrt[3]{\sum_{i} \sum_{k} x_{i, k}}$ The rest of the configurations can be seen in Table 1.

\subsection{Results and Analysis}

The idea behind the simulations is to show how the uniform auctioning framework performs in a practical scenario, highlight some of the foreseen problems and how it can be solved. First, what happens if the infrastructure provider does not set any reserved price. Since the resources are homogeneous (in the perspective of the infrastructure provider) and they are soled by a uniform price determined by the lowest winner price, there is a possibility that the virtual operators try to exploit this by reducing their bidding prices in order to maximize their profit. This could be seen in Figure 3, where virtual operator 1 manipulates the price and maximizes his profit (blue curve of the figure). Since the infrastructure provider has no reserved price set in that scenario, the virtual operator succeeds in reducing the resource price and thus increasing his profit. On the other hand, in the 2nd scenario (red curve of the figure) even though the virtual operator tries to manipulate his bidding price he is unable to increase his profit. This

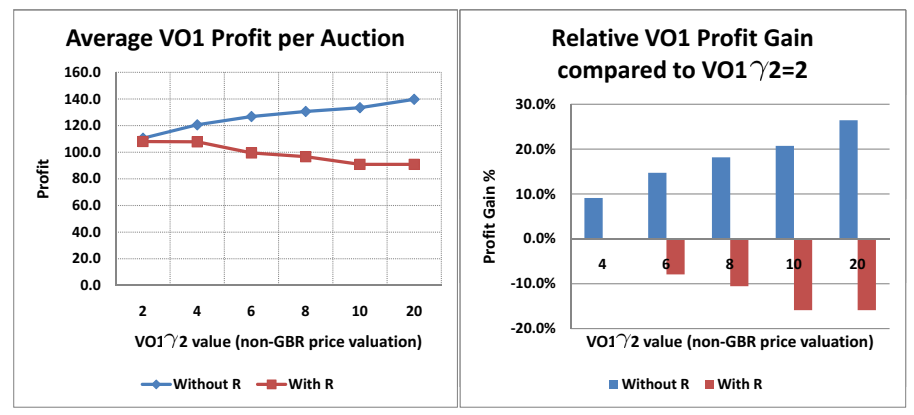

Fig. 3. Virtual Operator 1 profit and relative profit gain 


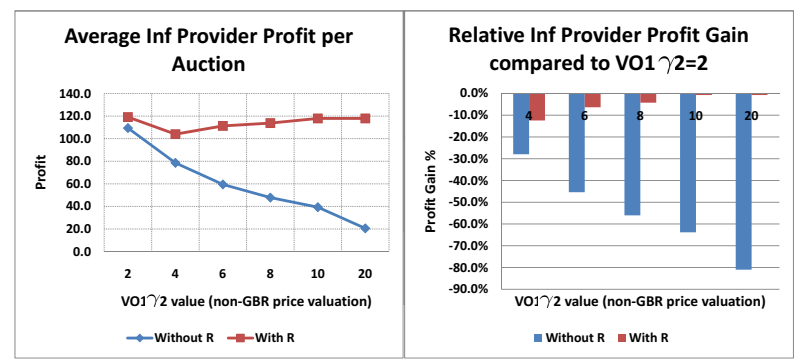

(a) Infrastructure provider (Auctioneer) profit and relative profit gain

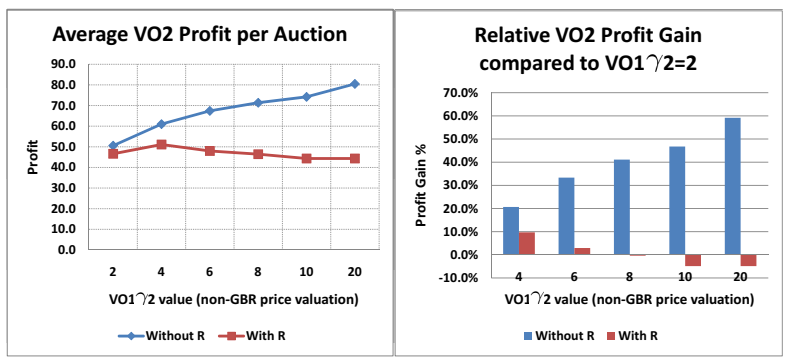

(b) Virtual Operator 2 profit and relative profit gain

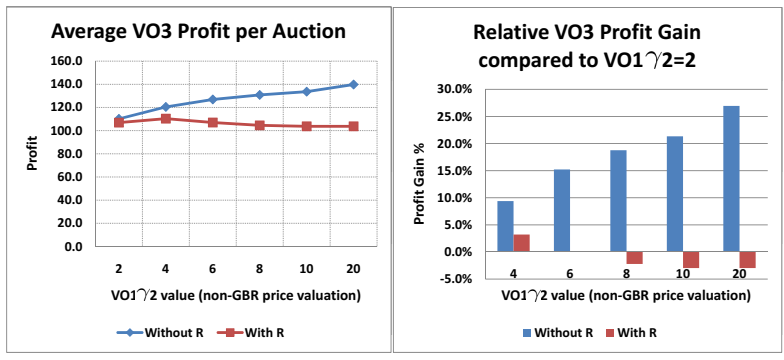

(c) Virtual Operator 3 profit and relative profit gain

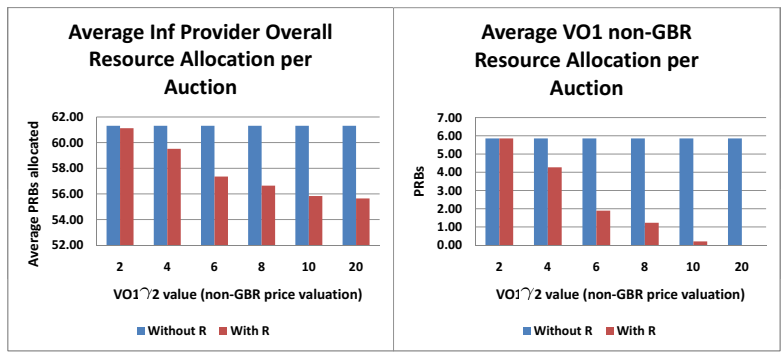

(d) Infrastructure provider and VO1 non-GBR overall resource utilization

Fig. 4. Figure presenting the profit and relative profit results and virtual operator resources 
is because the infrastructure provider sets a reserved price that the operator can not bid below. Figure 4a shows the infrastructure provider profit. The profit decreases in the case with no reserved price due to the virtual operator's price manipulation, but when the infrastructure provider sets a reserved price he can stop the operator manipulation. Figure $4 \mathrm{~b}$ and $4 \mathrm{c}$ show the average profit of virtual operator 2 and 3. The results are similar to virtual operator 1 results. The average number of PRBs (spectrum utilization) for both the infrastructure provider as well as virtual operator 1 can be seen in Figure 4d. Similar trend as before can be observed, in the scenario with no reserved price the amount of resources is the same in all cases because the virtual operator gets all of his demand but with different prices. In the scenario with a reserved price it can be seen that the overall resources granted to the virtual operator starts decreasing, this is because when the virtual operator starts manipulating the bidding price he will not be granted his demand if the price is lower than the reserved one.

\section{Conclusion}

In this paper we discussed dynamic infrastructure resource auctioning in future wireless paradigm by focusing on both technical and theoretical aspects. We provide the technical realization of the proposed future market behaviour with the state-of-the-art technical solution on the granule level. We have investigated how the uniform auctioning format performs when used to trade the spectrum between different virtual operators in a virtualized LTE system environment. Different scenarios were investigated, mainly what happens if the infrastructure provider does not set any reserved price and how the virtual operators can be stopped from manipulating the bids to maximize his profit. We tend to extend this work and investigate more on the potential economical and technical issues.

\section{References}

1. Bavier, A., Bowman, M., Culler, D., Chun, B., Karlin, S., Muir, S., Peterson, L., Roscoe, T., Spalink, T., Wawrzoniak, M.: Operating system support for planetary-scale network services (March 2004)

2. Paul, S., Seshan, S.: Geni technical document on wireless virtualization (September 2006)

3. AKARI architecture conceptual design for new generation network

4. Bauck, S., Görg, C.: Virtualisation as a co-existence tool in a future internet. In: ICT Mobile Summit - 4WARD Workshop, Stockholm, Sweden (June 2008)

5. Zaki, Y., Zhao, L., Timm-Giel, A., Görg, C.: A Novel LTE Wireless Virtualization Framework. In: Second International ICST Conference on Mobile Networks And Management (Monami), Santander, Spain, pp. 1-13 CD publication (September 2010)

6. Zaki, Y., Zhao, L., Timm-Giel, A., Görg, C.: LTE Wireless Virtualization and Spectrum Management. In: Third Joint IFIP Wireless and Mobile Networking Conference (WMNC), Budapest, Hungary (October 2010)

7. DARPA Next Generation Communication Program, http: / / www . sharedspectrum. com/resources / darpa-next-generation-communications-program

8. Buddhikot, M.M., Kolodzy, P., Miller, S., Ryan, K., Evans, J.: Dimsumnet: New directions in wireless networking using coordinated dynamic spectrum access. In: IEEE WoWMoM 2005, pp. 78-85 (2005) 
9. Rodriguez, V., Moessner, K., Tafazolli, R.: Market-driven dynamic spectrum allocation: Optimal end-user pricing and admission control for cdma. In: The Proceedings of IST Mobile and Wireless Communication Summit (2005)

10. Rodriguez, V., Moessner, K., Tafazolli, R.: Auction driven dynamic spectrum allocation: optimal bidding, pricing and service priorities for multi-rate, multi-class cdma. In: The Proceedings of 16th Internationalymposium on Personal, Indoor and Mobile Radio Communications (PIMRC), pp. 1850-1854 (2005)

11. Subramanian, A.P., Al-Ayyoub, M., Gupta, H., Das, S.R., Buddhikot, M.M.: Near-optimal dynamic spectrum allocation in cellular networks. In: 3rd IEEE Symposium on New Frontiers in Dynamic Spectrum Access Networks, DySPAN, pp. 1-11 (2008)

12. Yang, L., Cao, L., Zheng, H.: Physical interference driven dynamic spectrum management. In: Proc. of IEEE DySPAN (2008)

13. Zhou, X., Gandhi, S., Suri, S., Zheng, H.: ebay in the sky: strategy-proof wireless spectrum auctions. In: Proceedings of the 14th ACM International Conference on Mobile Computing and Networking, MobiCom 2008, pp. 2-13. ACM, New York (2008)

14. Sorabh, G., Buragohain, C., Cao, L., Zheng, H., Suri, S.: A general framework for wireless spectrum.

15. Sengupta, S., Chatterjee, M., Ganguly, S.: An economic framework for spectrum allocation and service pricing with competitive wireless service providers. In: 2nd IEEE International Symposium on New Frontiers in Dynamic Spectrum Access Networks, DySPAN, pp. 89-98 (2007)

16. Cramton, P.: The efficiency of the fcc spectrum auctions. Journal of Law and Economics 41 (October 1998)

17. Spectrum auctions. Auction design issues for spectrum awards market analysis Ltd.

18. French, R.: Spectrum auctions 101. The Journal of Public Sector Management (2008)

19. Sandholm, T., Suri, S.: Market clearability. In: Proceedings of the Seventeenth International Joint Conference on Artificial Intelligence, pp. 1145-1151 (2001)

20. Williams, D.E., Garcia, J.: Virtualization with $\mathrm{Xen}^{\mathrm{TM}}$ : Including XenEnterprise ${ }^{\mathrm{TM}}$, XenServer $^{\mathrm{TM}}$, and XenExpress ${ }^{\mathrm{TM}}$. In: SYNGRESS 2007 (2007)

21. OPNET, http: //www. opnet. com 